Kali P. Rath, Department of Economics, University of Notre Dame,

Notre Dame, IN 46556, USA, email: rath.1@@nd.edu

\title{
THE NONCONVERGENCE OF A CLASS OF MEASURABLE FUNCTIONS
}

\begin{abstract}
Let $\left\{\epsilon_{n}\right\}$ be a sequence of positive numbers converging to 0 . For each $n$ divide the unit interval $[0,1]$ into sub-intervals of the type $\left[k \epsilon_{n}\right.$, $\left.(k+1) \epsilon_{n}\right), k=0,1,2, \ldots$ and define a function $f^{n}$ as $1,-1,1,-1, \ldots$ successively on these intervals. If $\left\{f^{n_{k}}\right\}$ is any subsequence of $\left\{f^{n}\right\}$ then the set of points at which $\left\{f^{n_{k}}\right\}$ converges has Lebesgue measure zero. This is a generalization of the well known analogous result for Rademacher functions.
\end{abstract}

\section{Introduction}

Often, in theory as well as in applications, it is required to prove the convergence or nonconvergence of a given sequence of measurable functions. The most well known example of nonconvergence is the sequence of Rademacher functions. These functions take only two values and the sequence converges nowhere on the unit interval. The purpose of this note, is to discuss a general class of such functions which exhibit the same property. The motivation to consider the general case comes from game theory.

\section{Rademacher Functions}

For $n=1,2, \ldots$, the $n$th Rademacher function $r^{n}$ is defined on the unit interval $[0,1]$ by

$$
r^{n}(t)=(-1)^{k} \text { if } \frac{k}{2^{n}} \leq t<\frac{k+1}{2^{n}}, \quad k=0,1, \ldots, 2^{n}
$$

Key Words: Rademacher functions, Lebesgue measure, almost everywhere convergence and nonconvergence

Mathematical Reviews subject classification: Primary: 28A20 Secondary: 26A39

Received by the editors December 1, 1993 
Intuitively, for every $n=1,2, \ldots$, divide the unit interval $[0,1]$ into $2^{n}$ intervals of equal length and define the function $r^{n}$ as $1,-1,1,-1$ successively on these intervals. Thus, $r^{1}$ is 1 on $[0,1 / 2)$ and -1 on $[1 / 2,1) . r^{2}$ is 1 on $[0,1 / 4) \cup[1 / 2,3 / 4)$ and -1 on $[1 / 4,1 / 2) \cup[3 / 4,1)$. A key aspect of these functions, among others, is the following well known result.

Proposition 1 Let $\left\{r^{n_{k}}\right\}$ be any subsequence of $\left\{r^{n}\right\}$. Then the set of points at which $\left\{r^{n_{k}}\right\}$ converges has Lebesgue measure zero.

\section{$3 \quad$ A General Class of Functions}

The $n$th Rademacher function $r^{n}$, is defined by dividing the unit interval $[0,1]$ into $2^{n}$ intervals, each of equal length $\left(1 / 2^{n}\right)$. As $n$ tends to infinity, the lengths of these intervals go to zero. Here, a generalization of Proposition 1 will be obtained by focusing on this aspect.

Proposition 2 Let $\left\{\epsilon_{n}\right\}$ be a sequence of positive numbers converging to 0 and $\left\{f^{n}\right\}$ a sequence of real valued measurable functions on the unit interval $[0,1]$ given by

$$
f^{n}(t)=(-1)^{k} \quad \text { if } k \epsilon_{n} \leq t<(k+1) \epsilon_{n}, \quad k=0,1,2, \ldots
$$

Let $\left\{f^{n_{k}}\right\}$ be any subsequence of $\left\{f^{n}\right\}$. Then the set of points at which $\left\{f^{n_{k}}\right\}$ converges has Lebesgue measure zero.

For any fixed $n$, if $t$ belongs to the interval $\left[k \epsilon_{n},(k+1) \epsilon_{n}\right)$ for an even integer $k$ then the function $f^{n}$ takes value 1 at $t$, otherwise it takes value -1 . If $\epsilon_{n}=\left(1 / 2^{n}\right)$ for each $n$ then the functions are the Rademacher functions. The assertion is that no subsequence of $\left\{f^{n}\right\}$ converges on a set of positive Lebesgue measure. The issue, therefore, is to show that the lim inf of a sequence of unions of uniformly spaced intervals, with gaps the same size as the intervals, is a set of measure zero if the lengths of the intervals approach 0 .

An elementary fact about intervals on the real line is used in the proof. Let $[a, b)$ and $[c, d)$ be two nondegenerate intervals on the real line and suppose that their intersection is nonempty. Then, the intersection is also a nondegenerate interval closed in the left and open at the right. This fact generalizes to any two families of finite number of such intervals, i.e., the nonempty intersection of any two collections of finite number of nondegenerate left closed and right open intervals is itself a finite collection of nondegenerate left closed and right open intervals. 
Proof. Since every subsequence is a sequence of the same type, it's enough to prove the result for the entire sequence. For any $t, f^{n}(t)$ takes at most two values, 1 and -1 . So, if the sequence $\left\{f^{n}(t)\right\}$ converges at any $t$, its limit will be 1 or -1 . So, it needs to be shown that the set of points at which the functions converge to either has Lebesgue measure zero.

Towards this end, for any $n$, let $A_{n}$ denote the set of points at which $f^{n}$ is 1. $A_{n}=\left\{t \in[0,1) \mid f^{n}(t)=1\right\} \subseteq \cup_{k=0}^{\infty}\left[2 k \epsilon_{n},(2 k+1) \epsilon_{n}\right)$. Let $B_{n}=\{t \in$ $[0,1) \mid f^{m}(t)=1$ for all $\left.m \geq n\right\}$. Then $B_{n}=\cap_{m \geq n} A_{m}$ and $\cup_{n=1}^{\infty} B_{n}$ is precisely the set of points where $\left\{f^{n}\right\}$ converges to 1 . If $n<n^{\prime}$ then $B_{n} \subseteq B_{n^{\prime}}$. So, $\lambda\left(\cup_{n=1}^{\infty} B_{n}\right)=\sup _{n} \lambda\left(B_{n}\right)$ where $\lambda$ denotes Lebesgue measure. Therefore, to show that $\lambda\left(\cup_{n=1}^{\infty} B_{n}\right)=0$, it suffices to show that $\lambda\left(B_{n}\right)=0$ for each $n$.

Consider first, $A_{m} \cap[a, b)$ where $m \geq 1$ and $[a, b) \subseteq[0,1) . \lambda\left(A_{m} \cap[a, b)\right)=$ $\lambda\left(A_{m} \cap[0, b)\right)-\lambda\left(A_{m} \cap[0, a)\right)$. Any $x \geq 0$ can be written as $x=2 n \epsilon_{m}+r$ where $n$ is a nonnegative integer and $0 \leq r<2 \epsilon_{m}$. So, $\lambda\left(A_{m} \cap[0, x)\right)=$ $n \epsilon_{m}+\min \left\{r, \epsilon_{m}\right\}=n \epsilon_{m}+(1 / 2)\left(r+\epsilon_{m}-\left|r-\epsilon_{m}\right|\right)=(x / 2)+(1 / 2) d_{x}$ where $d_{x}$ is the distance between $x$ and the set $\left\{2 k \epsilon_{m} \mid k=0,1,2, \ldots\right\}$.

Therefore, $\lambda\left(A_{m} \cap[a, b)\right)=[(b-a) / 2]+(1 / 2)\left[d_{b}-d_{a}\right]$, which gives $[(b-$ a)/2] $-(1 / 2) d_{a} \leq \lambda\left(A_{m} \cap[a, b)\right) \leq[(b-a) / 2]+(1 / 2) d_{b}$. Equality can hold on either side. $d_{a} \leq \epsilon_{m}$ and $d_{b} \leq \epsilon_{m}$, yield the estimates $[(b-a) / 2]-(1 / 2) \epsilon_{m} \leq$ $\lambda\left(A_{m} \cap[a, b)\right) \leq[(b-a) / 2]+(1 / 2) \epsilon_{m}$.

If one further assumes that, $\epsilon_{m} \leq(1 / p)(b-a)$ for an integer $p>1$, then $A_{m} \cap[a, b)$ is nonempty and $\lambda\left(A_{m} \cap[a, b)\right) \leq(b-a)[(1 / 2)+(1 / 2 p)] \leq$ $(3 / 4)(b-a)$.

That $\lambda\left(B_{n}\right)=0$ for each $n$ can be easily established now. Let $0 \leq \delta_{n}=$ $\inf _{F}\left\{\lambda\left(\cap_{i \in F} A_{i}\right)\right\}$ where the infimum is taken over all nonempty finite subsets $F$ of $\{n, n+1, \ldots\}$. $B_{n}$ is a subset of $\cap_{i \in F} A_{i}$ for any such $F$, so, $\lambda\left(B_{n}\right) \leq \delta_{n}$. Therefore, to show that $\lambda\left(B_{n}\right)=0$, it suffices to show that $\delta_{n}$ is zero.

Suppose to the contrary. Then for some $F, 0<\delta_{n} \leq \lambda\left(\cap_{i \in F} A_{i}\right)<(4 / 3) \delta_{n}$. Obviously, $\cap_{i \in F} A_{i}$ is nonempty. For any $i, A_{i}$ is a finite union of pairwise disjoint nondegenerate intervals, closed in the left and open at the right. Since $F$ is finite and $\cap_{i \in F} A_{i}$ is nonempty, $\cap_{i \in F} A_{i}$ is a finite union of pairwise disjoint nondegenerate intervals. Let $\theta_{n}$ be the minimum length of the constituent intervals of $\cap_{i \in F} A_{i}$. Since these intervals are nondegenerate and there are finitely many of them, $\theta_{n}>0$. Choose an integer $m$ such that $m>\sup F$ and $\epsilon_{m} \leq(1 / p) \theta_{n}$ for some integer $p>1$. Such an $m$ exists because $F$ is finite and $\left\{\epsilon_{k}\right\} \rightarrow 0$.

Let $P$ be any of the constituent intervals of $\cap_{i \in F} A_{i}$. Then $\epsilon_{m} \leq(1 / p) \lambda(P)$. The arguments given above imply that $\lambda\left(A_{m} \cap P\right) \leq[(1 / 2)+(1 / 2 p)] \lambda(P) \leq$ $(3 / 4) \lambda(P)$. Taking the union over all the constituent intervals $P$ contained in $\cap_{i \in F} A_{i}$ yields that $\lambda\left(\left[\cap_{i \in F} A_{i}\right] \cap A_{m}\right) \leq(3 / 4) \lambda\left(\cap_{i \in F} A_{i}\right)<\delta_{n}$. This contradic- 
tion establishes that $\delta_{n}=0$ which implies that $\lambda\left(B_{n}\right)=0$ for any $n$. So, the set of points at which the sequence of functions converges to 1 has Lebesgue measure zero.

An analogous argument can be used to show that the set of points where $\left\{f^{n}\right\}$ converges to -1 has Lebesgue measure zero. The obvious modification required above is to redefine $A_{n}$ for each $n$ as the set of points at which $f^{n}$ is -1 . $A_{n}=\left\{t \in[0,1) \mid f^{n}(t)=-1\right\} \subseteq \cup_{k=0}^{\infty}\left[(2 k+1) \epsilon_{n},(2 k+2) \epsilon_{n}\right)$. Let $B_{n}=\left\{t \in[0,1) \mid f^{m}(t)=-1\right.$ for all $\left.m \geq n\right\}$. Then $B_{n}=\cap_{m \geq n} A_{m}$ as before and $\cup_{n=1}^{\infty} B_{n}$ is the set of points where $\left\{f^{n}\right\}$ converges to -1 . To estimate $\lambda\left(A_{m} \cap[a, b)\right)$, take $x$ and $d_{x}$ as before. $\lambda\left(A_{m} \cap[0, x)\right)=n \epsilon_{m}+\max \left\{0, r-\epsilon_{m}\right\}=$ $n \epsilon_{m}+(1 / 2)\left(r-\epsilon_{m}+\left|r-\epsilon_{m}\right|\right)=(x / 2)-(1 / 2) d_{x}$. So, $\lambda\left(A_{m} \cap[a, b)\right)=$ $[(b-a) / 2]-(1 / 2)\left[d_{b}-d_{a}\right]$ which yields $\lambda\left(A_{m} \cap[a, b)\right) \leq[(b-a) / 2]+(1 / 2) \epsilon_{m}$. Proceeding as before, one can conclude that the set of points at which $\left\{f^{n}\right\}$ converges to -1 has Lebesgue measure zero.

Therefore, the set of points at which any given subsequence of functions converges has Lebesgue measure zero. This completes the proof.

Acknowledgement. My greatest debt is to Thomas E. Armstrong and Neerchal K. Nagaraj for many stimulating conversations and insights. Comments from M. Ali Khan and Thomas Mathew have been extremely useful. Thanks are also due to the referee, whose suggestions led to many improvements in the proof. 